\title{
Smart window for angular selective filtering of solar radiation
}

\author{
$R$. Zakirullin ${ }^{1, *}$, and $I$. Odenbakh ${ }^{1}$ \\ ${ }^{1}$ Orenburg State University, Orenburg, Russia
}

\begin{abstract}
A new approach to angular selective filtering of the solar radiation without using the sunlight redistribution devices is proposed. Parallel strips of chromogenic materials on two surfaces of the pane(s) form an optical filter having angular selective light transmission. Clarified methods to calculate the optimum slope angle of the strips on the pane(s), their widths and relative position on two surfaces considering the seasonal and daily change in the solar radiation, the location of the building and the window's azimuth are presented. Such a smart window blocks the direct radiation in a preset angular range and transmits the scattered and reflected radiation that is provides comfortable daylighting indoors.
\end{abstract}

\section{Introduction}

Chromogenic materials potentially applicable in the smart windows for filtering solar radiation have a variety of mechanisms for changing the light transmission depending on change in the ambient conditions or under the influence of electric current. Chromogenics based on the transition metal oxide thin films have been discussed in [1]. Transmittance of the photochromic glass depends on the intensity of shortwave range of the solar radiation spectrum [2, 3]. Thermochromic materials control the solar radiation indoors [4], and thermotropic materials change their light scattering properties dependently on the temperature [5]. Optical properties of gasochromics [6] vary when reacting with hydrogen in the air. The application of electrical voltage changes the transmittance of electrochromic glasses [7, 8]. Smart glasses with liquid crystals [9] and suspended particles [10] are transparent when the electrical voltage is applied, without voltage they are opaque.

Advanced architectural glass coatings are an important component to improve the energy efficiency of the buildings [10-12]. Smart glasses currently used in the windows regulate the temperature, insolation and illumination level indoors, but cannot provide the angular selective light transmission of the window dynamically adapted to the position of the sun. Such a function requires the use of the blinds or static angular selective shading systems [13]. Chromogenic coating of a conventional smart window covers its entire area and attenuates not only direct, but also scattered and reflected radiation.

The objective of this study is expansion of the functionality of smart windows by using the chromogenic materials in novel optical filter [14-16] with the angular selective light transmission. Such a filter blocks the direct radiation partially or completely in a preset angular range (at the position of the sun) and transmits the scattered and reflected radiation. The methods to calculate the filter parameters given in [1416] are clarified.

\section{Methods}

An optical filter presented in [14-16] consists of thin film grating layers on two surfaces of the single or double glazed window. The input and output gratings are formed by non-transmissive parallel strips alternating with directionally transmissive strips. The nontransmissive (absorptive, reflective, or scattering) strips may hinder the view through the window and can be applied to vertical windows not used for viewing, to inclined or curved windows and skylights. To ensure sufficient visibility it is more preferable to create the non-transmissive strips by the photochromic, thermochromic, electrochromic or other smart technologies. In this case, the grating filter having all the advantages of smart glass will display its properties only with the appropriate change in the environmental conditions or under the influence of electric current, providing the view through the window in other cases.

Figure 1 shows a grating filter, alternating transmissive and chromogenic strips of which are sloped to be adapted to the sun's trajectory and located inside the panes. Active gratings with chromogenic strips can also be located on the inner surfaces of the window chamber in the form of thin film surface coatings or on both surfaces of the single glazed window [14-16]. The characteristic angle of the filter $\Theta_{c}$ determined by tracing of the beam passing through the centers of the strips of both gratings shows their shift $\delta$ relative to each other at distance $s$ between gratings. Proportion of the passing direct sunlight depends on the incidence angle $\Theta$ (figure 1). This method of angular regulation of the light transmission is patented by the author (RU Patents 2509324 and 2677069). The theoretical angular characteristic of the filter consists of alternating areas

* Corresponding author: rustam.zakirullin@gmail.com 
with decreasing, minimum, increasing and maximum light transmittance [14-16].
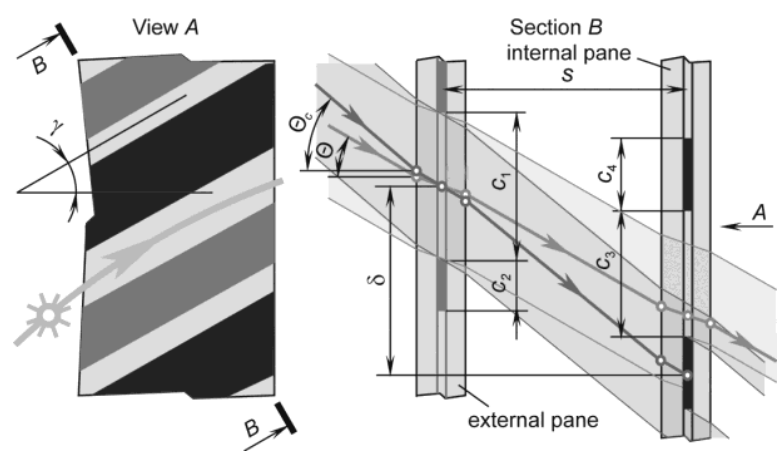

Fig. 1. An optical filter with sloped gratings adapted to the sun's trajectory. $\Theta$ is incidence angle, $\Theta_{\mathrm{c}}$ is characteristic angle of the filter, $s$ is distance between gratings, $\delta$ is shift of gratings relative to each other, $\gamma$ is slope angle of the filter's gratings, $c_{1}$ and $c_{3}$ are widths of the transmissive strips, $c_{2}$ and $c_{4}$ are widths of the chromogenic strips.

The optimum slope angle of filter's gratings for a window with the given azimuth can be determined according to an algorithm [16] including "1) determination of the elevation and azimuth of the sun relative to the specified window, 2) plotting the trajectories of the output surface trace of the point of incidence of the solar beams and the isochrones with identical time for the days of equinoxes and solstices, 3) determination of the optimum slope angle of the filter gratings for date and time with the maximal sun protection requirements". Here we present a new more effective method to determine the optimum slope angle of filter's gratings.

1) The calculation date is selected taking into account the local climate. As such a date, the middle of the hottest period of the year or the day of maximum solar radiation can be taken.

2) Considering the geographical coordinates of the building, elevation $h$ and azimuth $A$ of the sun are calculated for the selected date through every minute (hour, etc.) relative to the time when the solar beams fall in the plane perpendicular to the window ( $y$-axis in figure 2).

3) The azimuth of the sun relative to the window is calculated by: $\alpha=A-A_{0}$.

4) The incidence angle of the solar beam is calculated by $\Theta=\arccos (\cos h \cos \alpha)$ taking into account first cosine theorem for the trihedral angle: $\cos \Theta=\cos h \cos \alpha$.

5) Coordinates of the trace of the sun's trajectory on vertical plane of the window are calculated as: $x=\tan \alpha$; $y=\tan h$. This trace is plotted in figure 2 .

6) Equation of the straight line $y=k x+b$ is found after linear approximation of the curved trace of the sun's trajectory (figure 2). Using $k=\tan \gamma$, desired optimal slope angle $\gamma$ of the filter's gratings is equal to: $\gamma=\arctan k$.

Coordinates of the output surface trace of the point of incidence (figures 1 and 3) for a single glazed window are determined by:

$$
\begin{aligned}
& x=s \tan \left[\alpha \arcsin (\sin \Theta / n) \Theta^{-1}\right] \\
& y=-\left\{s \tan \left[\arcsin (\sin \Theta / n)-\left|\alpha \arcsin (\sin \Theta / n) \Theta^{-1}\right|\right] /\right. \\
& \left./ \cos \left[\alpha \arcsin (\sin \Theta / n) \Theta^{-1}\right]\right\}
\end{aligned}
$$

where $n$ is the refractive index of the glass. For a double glazed window:

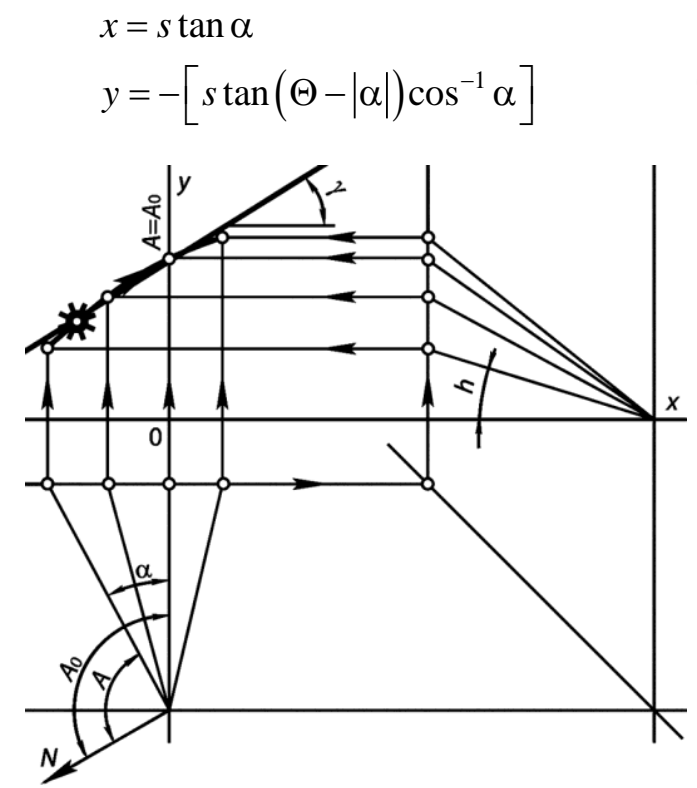

Fig. 2. Determination of the sun's trajectory relative to window and slope angle of filter's gratings on the window pane. $A$ is azimuth of the sun, $A_{0}$ is the window's azimuth, $\alpha$ is azimuth of the sun relative to the window, $h$ is elevation of the sun, $\gamma$ is slope angle.

For coordinates $x$ and $y$ calculated by equations (1) or (2), the characteristic angle of the filter is determined, at which the light transmittance of the window will have the minimum value (when analyzing figures 1 and 3 ):

$$
\begin{aligned}
& \Theta_{c}=\arctan \left(\delta s^{-1}\right)=\arctan \left(\left\{\sqrt{x^{2}+y^{2}} .\right.\right. \\
& \left.\cdot \cos [\gamma-\arctan (x / y)]\} s^{-1}\right)
\end{aligned}
$$

The minimum and maximum real (required) values of the light transmittance are set in the ranges: $0 \leq \tau_{\text {rmin }} \leq$ 0.5 and $0.3 \leq \tau_{\text {rmax }} \leq 0.88$ (for single glazed window), 0.3 $\leq \tau_{\text {rmax }} \leq 0.8$ (for double glazed window), $0.3 \leq \tau_{\text {rmax }} \leq$ 0.72 (for triple glazed window, in this case, one of two window chambers is used for the filter as in figure 1, Section $B$ ). The upper bounds of the ranges are conditional because of physical limitations. The minimum transmittance can be achieved at the preset time (for example, at the time of maximum solar radiation) or in some time interval around it.

The widths of strips of the filter's gratings $c_{1}, c_{2}, c_{3}$ and $c_{4}$ are determined from a system of four equations, the first of which is the equality of sums of the widths of strips of two gratings (figure 1): 


$$
c_{1}+c_{2}=c_{3}+c_{4}
$$

The minimum and maximum theoretical light transmittances are equal to:

$$
\begin{aligned}
& \tau_{\min }=\left(c_{3}-c_{2}\right) /\left(c_{1}+c_{2}\right) \\
& \tau_{\max }=c_{3} /\left(c_{1}+c_{2}\right), \text { if } c_{1}>c_{3},
\end{aligned}
$$

where $\tau_{\min }$ and $\tau_{\max }$ are calculated taking into account the reflection according to Fresnel equations, as well as the absorption according to Bouguer-Lambert law:

$$
\begin{aligned}
& \tau=\tau_{\mathrm{r}} /\left[1-0.5\left(\frac{\sin ^{2}[\Theta-\arcsin (\sin \Theta / n)]}{\sin ^{2}[\Theta+\arcsin (\sin \Theta / n)]}+\right.\right. \\
& \left.\left.\frac{\tan ^{2}[\Theta-\arcsin (\sin \Theta / n)]}{\tan ^{2}[\Theta+\arcsin (\sin \Theta / n)]}\right\}\right) . \\
& \cdot \exp \left[-\alpha_{\mathrm{a}} s\left(\frac{1+\sin ^{2} \Theta}{n^{2}-\sin ^{2} \Theta}\right)^{1 / 2}\right]
\end{aligned}
$$

where $\tau_{\mathrm{r}}$ is the preset real light transmittance; $\Theta$ is the incidence angle, deg; $\alpha_{\mathrm{a}}$ is the natural absorptance of the glass, $\mathrm{mm}^{-1} ; s$ is the total thickness of all panes, $\mathrm{mm}$. The exponent of first multiplier in the denominator is 2,4 and 6 for a single, double and triple glazed windows, respectively. For average value of the light transmittance $\left(\tau_{\mathrm{av}}=0.5\left(\tau_{\min }+\tau_{\min }\right)\right)$ at a specified average angle $\Theta_{\mathrm{av}}$ $\left(\Theta_{\mathrm{av}}<\Theta_{\mathrm{c}}\right)$, fourth equation is obtained for the single or double/triple glazed window at the distance $s$ between gratings, respectively:

$$
\begin{aligned}
& 0.5\left(\tau_{\min }+\tau_{\max }\right)=\left[0.5 c_{1}-0.5 c_{4}+\right. \\
& +s \sin \Theta_{c}\left(\sqrt{n^{2}-\sin ^{2} \Theta_{c}}\right)^{1 / 2}- \\
& \left.-s \sin \Theta_{a v}\left(\sqrt{n^{2}-\sin ^{2} \Theta_{a v}}\right)^{1 / 2}\right]\left(c_{1}+c_{2}\right)^{-1} ; \\
& 0.5\left(\tau_{\min }+\tau_{\max }\right)=\left(0.5 c_{1}-0.5 c_{4}+\right. \\
& \left.+s \tan \Theta_{c}-s \tan \Theta_{a v}\right)\left(c_{1}+c_{2}\right)^{-1} .
\end{aligned}
$$

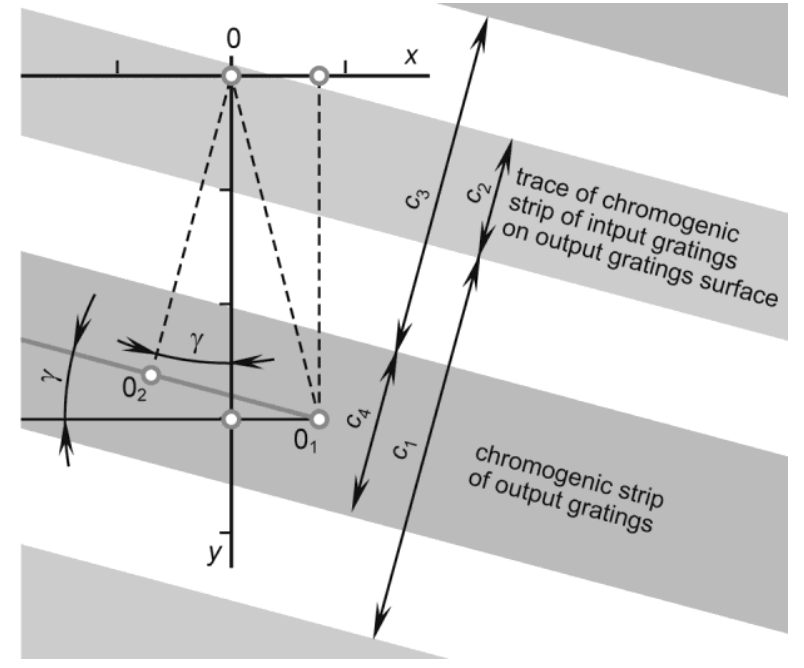

Fig. 3. Determination of characteristic angle of the filter. 0 is the incidence point on the filter's input gratings surface, $0_{1}$ is the trace of the point 0 on the filter's output gratings surface at the characteristic angle, $0_{2}$ is the projection of the point $0_{1}$ on the plane perpendicular to the strips, line segment $00_{2}$ is shift between filter's gratings.

Solving the system of equations (4)-(6) and (8) gives the values of the widths of strips (the first two equations are for the single and double/triple glazed windows, respectively):

$$
\begin{aligned}
& c_{3}=2 s \sin \Theta_{c}\left(\sqrt{n^{2}-\sin ^{2} \Theta_{c}}\right)^{1 / 2}- \\
& -2 s \sin \Theta_{a v}\left(\sqrt{n^{2}-\sin ^{2} \Theta_{a v}}\right)^{1 / 2} ; \\
& c_{3}=2 s \tan \Theta_{c}-2 s \tan \Theta_{a v} ; \\
& c_{4}=c_{3}\left(1-\tau_{\max }\right) \tau_{\max }^{-1} ; \\
& c_{2}=c_{3}-\tau_{\min }\left(c_{3}+c_{4}\right) ; \\
& c_{1}=c_{3}+c_{4}-c_{2} .
\end{aligned}
$$

\section{Calculation}

Theoretical methods to determine the optimum slope angle of filter's gratings, the characteristic angle of filter and the widths of strips given in Section 2 are the basis to achieve a preset angular characteristic of the light transmission of the smart window considering its azimuth.

After determining the parameters of the filter, its theoretical angular characteristic is calculated. The areas with the constant minimum and maximum transmittance are calculated by equations (5) and (6), respectively. The decreasing and increasing areas are calculated by:

$$
\tau=\left(|\Delta|-0.5 c_{2}+0.5 c_{3}\right)\left(c_{1}+c_{2}\right)^{-1},
$$

where $\Delta$ is the shift between the traces of the input gratings on the surface of the output gratings at the characteristic angle and an arbitrary incidence angle (the shift between the point $0_{2}$ in figure 3 and a similar point for the arbitrary incidence angle) calculated as: 


$$
\begin{aligned}
& \Delta=\sqrt{x_{\min }^{2}+y_{\min }^{2}} \cos \left[\gamma-\arctan \left(x_{\min } / y_{\min }\right)\right]- \\
& -\sqrt{x^{2}+y^{2}} \cos [\gamma-\arctan (x / y)] .
\end{aligned}
$$

Finally, values of the real light transmittance $\tau_{\mathrm{r}}$ are expressed from equation (7) substituting the theoretical light transmittance calculated by equations (5), (6) and (10).

\section{Results and discussion}

Table 1 shows the results of numerical calculations of the slope angles of filter's gratings for four azimuths of the windows according to the algorithm given in [16] and the new algorithm proposed in Section 2. The slope angles are determined for the city of Orenburg, Russia (the latitude of $52.28^{\circ}$, the longitude of $55.17^{\circ}$, GMT+05:00), on 15.07.2013 (for the middle of the hottest period in the city of Orenburg), at a distance between gratings $s=4 \mathrm{~mm}$ and refractive index $n=1.5$ [16].

Table 1. The slope angles of filter's gratings for the different azimuths of the windows. The angles are determined by the algorithm given in [16] and the proposed new algorithm.

\begin{tabular}{|c|c|c|}
\hline \multirow{2}{*}{$\begin{array}{c}\text { Azimuth of } \\
\text { window (grad) }\end{array}$} & \multicolumn{2}{|c|}{ Slope angle (grad) } \\
\cline { 2 - 3 } & {$[\mathbf{1 6}]$} & New \\
\hline 90 & 35 & 42 \\
\hline 120 & 30 & 38 \\
\hline 150 & 18 & 30 \\
\hline 180 & 0 & 0 \\
\hline
\end{tabular}

The slope angles are determined by new algorithm at all same parameters except the distance between gratings and refractive index since the angles do not depend on them. The slope angles determined by the new algorithm are universal and suitable for windows with any glazing (by [16], the angles for the single and double glazed windows are different), as well as for devices with sloped shading elements, for example, for zebra blinds with sloped strips. The slope angles obtained by the new algorithm are greater (table 1). For the south window with the azimuth of $180^{\circ}$, the angles are the same since the sun's trajectory in this case is symmetric with respect to the zenith position of the sun.

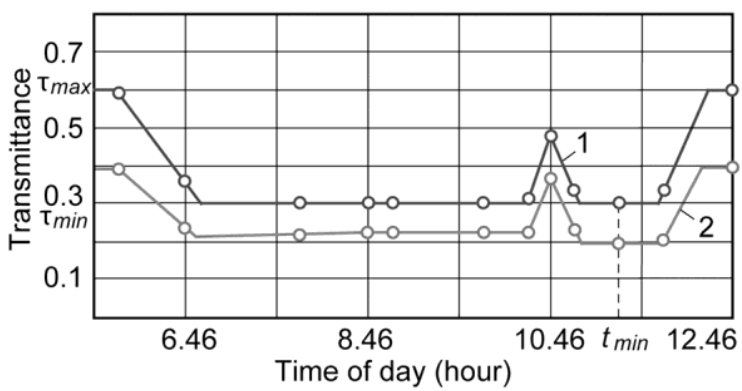

Fig. 4. Theoretical (1) and real (2) angular characteristics of the light transmittance of the filter.
The characteristic angle of the filter is determined for the double glazed window with azimuth of $120^{\circ}$ at the distance between gratings $s=16 \mathrm{~mm}$ (figure 1). By table 1 , the slope angle is $38^{\circ}$. At $11 \mathrm{hr}$. $30 \mathrm{~min}$. (the time of maximum solar radiation in the city of Orenburg) the azimuth of the sun is $133.46^{\circ}$ and the elevation is $52.01^{\circ}$. At this time, the filter must provide the minimum light transmittance of the window. For $h=52.01^{\circ}$ and $\alpha=13.46^{\circ}$, the incidence angle is $\Theta=53.23^{\circ}$. For the coordinates $x=3.8294 \mathrm{~mm}$ and $y=-13.6979 \mathrm{~mm}$ (by equations (2)), the characteristic angle of the filter is $\Theta_{c}$ $=27.8^{\circ}$ (by equation (3)). The azimuth of the sun $A$ and the azimuth $A_{0}$ of the window are equal at $10 \mathrm{hr}$. $46 \mathrm{~min}$.

The widths of the strips are determined for the minimum and maximum required light transmittances $\tau_{\text {rmin }}=0.2$ (must be achieved in the time interval around $11 \mathrm{hr} .30 \mathrm{~min}$.) and $\tau_{\mathrm{rmax}}=0.4$ at the average incidence angle $\Theta_{\mathrm{av}}=10^{\circ}$. The minimum and maximum theoretical transmittances calculated by equation (7) at the natural absorptance of the glass $\alpha_{\mathrm{a}}=0.01 \mathrm{~mm}^{-1}$ are: $\tau_{\min }=0.3$ and $\tau_{\max }=0.6$. The widths of the strips calculated by equation (9) are equal: $c_{1}=13.1007, c_{2}=$ $5.6146, c_{3}=11.2292, c_{4}=7.4861 \mathrm{~mm}$.

The theoretical angular characteristic of the filter with determined parameters is presented in figure 4 by line 1. The maximum, decreasing, minimum and increasing light transmittance areas are calculated according to Section 3. The real characteristic (line 2) is calculated using equation (7) at the real incidence angle calculated by $\Theta=\arccos (\cos h \cos \alpha)$ for the corresponding time.

The results of the study demonstrate the real possibility to reach the minimum light transmittance of the smart window at the preset date and time of day. Table 1 comparing the previous and new calculations shows the need for a variety of the slope angles of filter's gratings for the different window orientations. The methods of determining the geometrical parameters of the filter considering all given local building conditions provide comfortable daylighting indoors. New approach to the use of chromogenic materials in smart windows expands their functionality.

\section{Conclusions}

We have presented the methods to determine the optimum slope angle of the filter's gratings on the window pane(s), the widths of the strips and their relative position on two surfaces considering the seasonal and daily change in the solar radiation, the location of the building and the window's azimuth. Unlike the conventional smart windows entire covered with chromogenic layer attenuating not only direct but also scattered and reflected radiation, the grating optical filter consisting of the chromogenic strips attenuates direct radiation and transmits comfortable scattered and reflected radiation. Such a smart window provides the angular selective light transmittance without the sunlight redistribution dev. 


\section{References}

[1] P. Ashrit, Transition Metal Oxide Thin FilmBased Chromogenics and Devices first ed (Amsterdam: Elsevier) (2017)

[2] H. Dürr and H. Bouas-.Laurent Photochromism: Molecules and Systems first ed (Amsterdam: Elsevier Science) (2003)

[3] M. Hocevar, S. Bogati, A. Georg, U. Opara Krasovec, Sol. Energy Mater. Sol. Cells 17185 (2017)

[4] B. Baloukas, S. Loquai, and L. Martinu, Sol. Energy Mater. Sol. Cells 183, 25-33 (2018)

[5] A.C. Gladen, J.H. Davidson, and S.C. Mantell, ASME J. Sol. Energy Eng. 137(2), 021003 (2014)

[6] K. Nishizawa, Y. Yamada, and K. Yoshimura, Sol. Energy Mater. Sol. Cells 170, 21-6 (2017)

[7] C.G. Granqvist, Handbook of Inorganic Electrochromic Materials first ed (Amsterdam: Elsevier Science) (1995)

[8] A. Piccolo, C. Marino, A. Nucara, and M. Pietrafesa, Energy and Buildings 165, 390-8 (2018)

[9] P.S. Drzaic, Liquid Crystals 33, (11-12) 1281-96 (2006)

[10] M. Casini, Smart Buildings: Advanced Materials and Nanotechnology to Improve EnergyEfficiency and Environmental Performance (Woodhead Publishing) (2016)

[11] U. Desideri, F.Asdrubali, Handbook of Energy Efficiency in Buildings first ed (ButterworthHeinemann) (2018)

[12] S.D. Rezaei, S. Shannigrahi, S. Ramakrishna, 2017 Sol. Energy Mater. Sol. Cells 159, 26-51

[13] L.L. Fernandes, E.S. Lee, A. McNeil, J.C. Jonsson, T. Nouidui, X. Pang, and S. Hoffmann, Energy and Buildings 90, 188-206 (2015)

[14] R.S. Zakirullin, Journal of Optical Technology 80(8), 480-5 (2013)

[15] R.S. Zakirullin, Appl. Opt. 54(21), 6416-19

[16] R.S. Zakirullin, S.N. Letuta, Solar Energy 120, 585-92 (2015) 